\title{
La Ilegalidad: una herramienta de análisis para la naturaleza del conflicto en Colombia
}

\author{
"La naturaleza humana es Guena y la maldad es \\ esencialmente antinatural" \\ Confucio
}

\section{RESUMEN}

Para muchos teóricos sobre la paz, encontrar respuestas al problema: ¿cómo superar la guerra? convergen múltiples áreas a su que hacer que pueden y pretenden dar respuesta a dicha pregunta, sin embargo una respuesta se encontraría en la naturaleza humana y en la misma naturaleza del conflicto. Para el caso colombiano, encontrar herramientas de análisis que lleven a la superación del conflicto, emplea elementos más allá de las políticas internas y la lucha del Estado frente a grupos ilegales, esta es la ilegalidad, que se encuentra por tanto dentro de la cultura de violencia definida por Johan Galtung. Desde estas disímiles teorías a las causas de la guerra, se puede afrontar la cuestión de cómo lograr una mayor paz en Colombia. De lo anterior el principal objetivo de este artículo no es construir un modelo para la paz, sino analizar elementos importantes que se dan dentro del conflicto y que son analizados bajo sucesos acometidos en ciertos momentos de la historia del conflicto colombiano. La principal conclusión es que en Colombia la naturaleza del conflicto prima por la cultura de violencia y la violencia que engendra junto con la ilegalidad en que también la sociedad basa su legitimidad junto con el narcotráfico, las pandillas, la insurgencia es hoy para el colombiano una salida fácil a los problemas económicos y sociales que enfrenta. 
PALABRAS CLAVE: conflicto, cultura de violencia, ilegalidad, violencia

\section{ABSTRACT}

For many theorists on peace, find answers to the problem: How to Overcome the war? Converge its multiple areas that can and do seek to answer that question, however a response would be in human nature and the nature of the conflict. For Colombia, find analysis tools that lead to ending the conflict, uses elements beyond the internal politics and the struggle of the state against illegal groups, this is illegal, which is therefore within the culture of violence as defined by Johan Galtung. From these disparate theories of the causes of war, we can address the issue of how to achieve greater peace in Colombia. From the above, the main objective of this article is to build a model for peace, but to analyze important elements that occur within the conflict and are analyzed from events undertaken at certain times in the history of the conflict. The main conclusion is that in Colombia the premium nature of conflict and violence culture that breeds violence with lawlessness in society also draws its legitimacy along with drug trafficking, gangs, insurgency in Colombia today for a easy way to economic and social problems it faces.

KEYWORDS: conflict, vulture of violence, illegality, violence

\section{INTRODUCCIÓN}

Charles Tilly en su libro sobre los Estados europeos, tipifica y argumenta como la guerra se convierte en motor para la conformación de dichas unidades políticas. La guerra como concepto indica el momento en el cual un conflicto pasa a un nivel de confrontación entre bandos cuyo objetivo es la derrota militar del otro o adversario, y en segunda instancia una posible negociación política para resolver el enfrentamiento. Atípico es entonces entender la formación de los Estados europeos sin este fenómeno, ¿pero esta razón es válida para entender la formación de los Estados latinoamericanos? Esta pregunta es demasiado amplia para responder en un trabajo como este pero si es necesario a partir 
de este ejercicio dar elementos sobre el tema. De tal manera el análisis se centra en revisar elementos teóricos sobre la naturaleza del conflicto e interpretar su utilidad para el conflicto colombiano. La naturaleza del conflicto refiere no simplemente a las causas que llevan a una confrontación de corto o largo aliento sino a las situaciones que transmiten latencia del mismo haciendo de los momentos de paz un simple apaciguamiento, así pues, estas situaciones de conflicto, responden a lugares de construcción política, económica, social y cultural de una sociedad. No fluyen de la nada son sentidos construidos por parte de actores precisos políticos que entienden que la vía del enfrentamiento también resuelve los distanciamientos entre posibilidades de un proyecto político.

A partir de lo anterior, la cultura de la violencia se ha convertido en una categoría de análisis que intenta reconstruir la naturaleza del conflicto colombiano, y este trabajo busca por lo tanto responder si las variables que sustentan el carácter de cultura de la violencia colombiana responden a la naturaleza del conflicto colombiano. Es necesario adelantar que el factor cultural es clave, los lugares de análisis de esta perspectiva si generan elementos precisos para el entendimiento de la naturaleza del conflicto colombiano. Sin embargo el cuestionamiento es la precisión y alcance de las variables que denotan que en Colombia hay una cultura de la violencia, y a partir de allí entender si esta condición explica suficientemente la naturaleza del conflicto.

El conflicto colombiano es el fenómeno latinoamericano que más se destaca dentro de la construcción del Estado, ya que dicho conflicto se encuentra irresuelto y no tiene posibilidades de una culminación a corto plazo, esto debido al redireccionamiento militar de actores precisos como la guerrilla y las llamadas bandas emergentes que son un reducto de los grupos paramilitares que entraron en un proceso de negociación para su desarticulación a través de la ley de justicia y paz durante el gobierno de Álvaro Uribe Vélez, proceso que logró por medio de la lucha electoral legitimar proyectos políticos liderados por los paramilitares en regiones de dominio político y económico que aun continúan a pesar del proceso de inserción de la vida civil. 
De lo anterior, este artículo pretende analizar un elemento del conflicto: la ilegalidad que intrínsecamente se encuentra ligada a la dinámica del conflicto, respondiendo a la pregunta que motivo a escribir estas páginas: ¿es la ilegalidad la base fundamental y única que genera la violencia en Colombia?

\section{Ilegalidad: elemento analítico para el conflicto}

El conflicto colombiano, ha tomado dimensiones que involucran a los Estados vecinos no como simple actores cooperantes con la institucionalidad colombiana para la resolución sino como afectados claramente por las dinámicas del fenómeno, tal es así que el compás de acción y relaciones se amplió con la muerte de un miembro del secretariado de las FARC Raúl Reyes en un bombardeo por parte del ejército colombiano en un campamento de dicha agrupación subversiva ubicado en territorio ecuatoriano. Es decir tal ataque es tan solo la punta del iceberg que denota que el conflicto colombiano se le salió de las manos a la institucionalidad colombiana.

Esta situación muestra muchas dinámicas para analizar, en un primer momento es como el conflicto colombiano desbordó las fronteras y por lo tanto la inexistencia de una política coordinada entre Colombia y los países vecinos, dejando entrever las dificultades suscitadas a partir de estos hechos. Sin embargo el tema de fondo y que los países de la región lograron identificar en tales acontecimientos es el lugar de la legalidad, es decir, como se defienden unos medios que sobrepasan el espacio de lo legal por un fin aparentemente supremo.

Por otra parte hay situaciones de carácter interno que empiezan a hacer eco aun en el plano internacional; tiene que ver con los seguimientos, interceptaciones telefónicas y medidas de seguridad del gobierno hecha por el Departamento Administrativo de Seguridad (DAS) cuya naturaleza le hace parte del ejecutivo y que muestra una serie de 
inconsistencias en las medidas y ejercicio de sus funciones que viola los derechos fundamentales y que atraviesa un proceso critico en el país que intenta esclarecer en el plano de la justicia.

A partir de lo anterior un primer momento de análisis es entender que a la violencia que referirá es a la violencia política, es decir la que se ejerce o tiene acción entre bandos en Colombia detectados claramente en la institucionalidad frente a la guerrilla y paramilitares, si se puede decir que hay un verdadero conflicto entre el Estado colombiano y paramilitares, por lo tanto allí observar una cultura de la violencia buscaría reconocer referentes de análisis que algunos teóricos de la cultura de la violencia en Colombia sostienen y aportar algunos otros en aspectos como lo históricamente constituido a lo largo de la dinámica del conflicto, esto es que hay una clara visión en Colombia y esta es que la violencia se constituye en la forma de solucionar las diferencias o conflictos. Reafirmando que esto no solo sucede en el plano político sino en esferas tan pequeñas que denotan como la violencia entre cercanos se ha convertido en una condición que genera los más altos índices en Colombia y más altos que aun la misma violencia política. Para el año 2009 una de las instituciones que cuenta con mayor aprobación y fiabilidad en Colombia -medicina legal- nombra que una de cada tres victimas que acude a la institución llegaron por lesiones generadas por un allegado, sumando casi 94.000 casos en donde riñas y venganzas personales están en el tope de causas de homicidio, las cifras revelan que cada hora suceden 10 ataques por lesiones entre allegados; fueron más de 13.000 casos de ataque sexual en el mismo año sucedidos entre los hogares de las mismas victimas, también aumento el asesinato de mujeres a manos de sus parejas. El año de 2009 dejo por lo tanto 1523 mujeres asesinadas 284 casos mas que el año anterior. $^{3}$

Sin embargo lo que la violencia política muestra es que no es tan precisa como parece, es decir esta permea y esta atravesada por diferentes conflictos que no es fácil generar

\footnotetext{
3Medicina Legal: "muestra la "anatomía" de la violencia colombiana" [en línea] disponible en la web: http://www.medicinalegal.gov.co/index.php?option=com_content\&view=article\&id=199\%3Amedicinalegal-muestra-la-anatomia-de-la-violencia-colombiana\&Itemid=144
} 
límites entre violencias, dígase el narcotráfico, pandillas juveniles, entre otras. Por otra parte lo que diferencia en cuanto al ojo del huracán, es que la violencia entre cercanos suma una a una sus víctimas pero la violencia política arrasa tanto uno a uno como en grandes números, la tasa nacional de homicidios supera el 70 por 100.000 habitantes y entre 1980 y 1995, el numero de los muertos de todo origen superó los 300.000 Según estudios del CINEP. Las masacres de 5 personas o más son innumerables solamente entre 1988 y 1993 se cuentan más de 900 de ellas que provocaron más de 5.000 víctimas. ${ }^{4}$

Esta tesis significaría una manera pragmática y posible de contestar si en Colombia existe una cultura de la violencia, sin embargo ¿Cómo explicar la latencia del conflicto mismo? ¿Es decir en que momentos puede observarse el ejercicio de esta condición? En primer momento hay que referir es que el papel de la ilegalidad se convierte en instrumento posible para lograr los fines de la institucionalidad. Por lo tanto por objeto de estudio preciso de este trabajo colocaremos a la institucionalidad dejando claro que los actores de la violencia en Colombia son múltiples.

Un recorrido simple pero necesario en este gobierno mirándolo como el actor que debe representar la institucionalidad y la legalidad muestra: 517 dirigentes sindicales asesinados durante el periodo de gobierno 2006-2010, o las artimañas para mantenerse en el poder "Yidispolítica", caso en el cual la representante a la Cámara Yidis Medina admitió haber recibido ofrecimientos de dádivas de parte de funcionarios del Gobierno Nacional a cambio de su voto favorable al proyecto de reforma constitucional que permitió que Álvaro Uribe Vélez aspirara a un segundo mandato presidencial; Si nos vamos a la división tripartita del poder paradigma democrático se observan actos del ejecutivo sobre la rama judicial como el espiar a magistrados o meter mafiosos al Palacio de Nariño para conspirar contra la Corte Suprema. Si se hace una lista indiscriminada se encuentra: seguimiento a periodistas y oponentes del gobierno; favorecer a ricos hacendados amigos y donantes a la campaña de Álvaro Uribe a la Presidencia de la República con subsidios

\footnotetext{
${ }^{4}$ Pecaut Daniel. Guerra contra la Sociedad. Editorial Planeta Colombia. Bogotá D.C. 2001. Pp. 187-190.
} 
que estaban dirigidos a campesinos a través del programa Agro Ingreso Seguro; intervenir y generar negociaciones para desarrollar proyectos empresariales de sus hijos con la complicidad de funcionarios complacientes; propiciar mediante recompensas materiales por bajas contadas situación que generó entre las filas del Ejército la comisión de más de 2.500 ejecuciones extrajudiciales "falsos positivos"; extraditar a la cúpula paramilitar cuando comenzó a prender el ventilador y empezaron a revelar sus vínculos con políticos colombianos e instituciones del Estado ; invadir y bombardear a un país extranjero (Ecuador) en busca de eliminar a su enemigo; usar emblemas de la Cruz Roja Internacional para obtener una victoria táctica con la "Operación Jaque"; defender en público -y con métodos privados- a los oficiales acusados por la Toma del Palacio de Justicia y los civiles torturados y desaparecidos durante el hecho; utilizar a alias 'Tasmania' para urdir un montaje contra el magistrado auxiliar a cargo de la parapolítica, Iván Velásquez; nombrar a Jorge Noguera en el DAS para lograr las medidas de usar la institucional para perseguir a la oposición; invitar a los parapolíticos a que no dejaran de apoyarlo con sus votos antes de que se los llevaran para la cárcel; repartir notarías y dádivas entre el Congreso en función de la segunda tentativa de reelección, etc. ${ }^{5}$ De lo anterior cabe hacer una odiosa comparación pertinente para el análisis, del caso; El escándalo de Watergate ${ }^{6}$, este caso y las acciones del anterior gobierno muestra cómo, si bien ambas fueron acciones ilegales dentro de contextos históricos diferentes, las

\footnotetext{
${ }^{5}$ www.semana.com Jorge Gómez Pinilla “Mockus en Peligro” Abril 28 de 2010.

${ }^{6}$ Watergate, comenzó con el arresto de cinco hombres por el allanamiento de la sede del Comité Demócrata Nacional en el complejo de oficinas Watergate, Las investigaciones llevadas a cabo por el FBI y después por el Comité de Watergate en el Senado, el House Judiciary Committee y la prensa revelaron que este robo fue sólo una de las múltiples actividades ilegales autorizadas y ejecutadas por el equipo de Nixon. También revelaron el enorme alcance de los crímenes y abusos, que incluían fraude en la campaña, espionaje político y sabotaje, instrucciones ilegales, auditorías de impuestos falsas, escuchas ilegales a gran escala, y un fondo secreto en México para pagar a quienes realizaban estas operaciones. Este fondo también se usó para comprar el silencio de los cinco hombres que fueron imputados por el robo del 17 de junio. Todo esto llevó a la renuncia de Nixon a la Presidencia de los Estados Unidos de América, toda la nación norteamericana, los líderes políticos y medios de comunicación se movilizo de varias maneras para tocar la conciencia nacional y expresar que como tal la nación no podía admitir la ilegalidad como medio para los fines del Estado.
} 
respuestas a los mismos zonas disímiles pero no precisamente lo histórico explica tal disparidad, ¿cuáles son las razones para tan dispar acción? Es decir que referentes se movilizan en circunstancias tan obvias de actos ilegítimos del Estado para que la sociedad y las mismas instituciones decidan actuar o no. Una posible respuesta es el relativizar los actos ilegítimos como apropiados en un momento donde el fin se hace supremo, pero la pregunta que surge es ¿cómo se logra esto?

Para Slavoj Zizek (2007) "la verdadera política, por tanto, trae siempre consigo una suerte de corto circuito entre el Universal y el Particular: la paradoja de un singulier universal, de un singular que aparece ocupando el Universal y desestabilizando el orden operativo "natural" de las relaciones en el cuerpo social (...) La verdadera lucha política, como explica Rancière contrastando a Habermas, no consiste en una discusión racional entre intereses múltiples, sino que es la lucha paralela por conseguir hacer oír la voz propia y que sea reconocida como la voz de un interlocutor legitimo". (pág. 25-27)

Colombia se encuentra en un momento de su historia donde se acomoda de manera clara y sin zonas grises, en un sentido político determinado por la "seguridad" es decir es un elemento que funciona como fuerza política homogenizadora. Desde al año 2002 y a partir de frustraciones políticas con respecto a la paz, el país intenta a través de un movimiento político radical enfrentar a los grupos armados con todo el peso de las instituciones cerrando o dificultando el paso a vías de negociación. La Seguridad Democrática, propuesta del programa de Álvaro Uribe Vélez tiene un campo que le materializa, el Estado Comunitario, campo que desarrolla otras dinámicas que no son objeto de reflexión por ahora. Sin embargo, en plena época electoral es claro observar que el tema de la Seguridad Democrática muy a pesar de todas las situaciones previamente enunciadas ocupa el centro de las propuestas políticas, ningún candidato presidencial se atreve a decir que va a negociar y mucho menos que la seguridad democrática fue un espejismo, que con las informaciones del Comité Internacional de la Cruz Roja se han fortalecidas, y por otra parte los paramilitares mutaron a bandas emergentes, es decir solo se transformaron algunas unidades de la cúpula donde los ordenes y objetivos siguen en los mismos controles territoriales económicos y políticos, y 
allí algunos reinsertados decidieron volver a la actividad delictiva por los réditos económicos que les ofrece pertenecer dichos grupos ilegales.

La Seguridad Democrática, cala en los odios de todos los colombianos y es resumida según el sentido común cómo la lucha contra las FARC, para el gobierno nacional se descifra así. Así Colombia enfrenta el desafío inmenso de la inseguridad interna que amenaza a la democracia; en respuesta, la política de seguridad democrática se diseñó como un instrumento para su defensa. La seguridad es un valor democrático y la paz depende de su preservación. La política de seguridad democrática es:

- Una política de recuperación de las libertades públicas, conculcadas por la acción terrorista de grupos armados por fuera de la ley, cuyo avance no ha sido debidamente confrontado por el Estado.

- Un compromiso de derrota de los violentos por parte del Estado.

- El ejercicio del legítimo derecho a la defensa propia que toda democracia tiene cuando se ve amenazada por la violencia terrorista.

La política de la Seguridad Democrática, por lo tanto, no es más que un puente hacia la construcción definitiva de la seguridad y la reconciliación nacional, que no es unanimismo, sino civilidad del debate, por medio de la defensa del pluralismo y el respeto a los procedimientos democráticos.

La política de Seguridad Democrática es un instrumento de recuperación de la seguridad con absoluto respeto a la democracia, a diferencia de las viejas doctrinas latinoamericanas de la seguridad nacional, que suprimieron las libertades, sustentaron dictaduras, eliminaron el disenso, descalificaron la oposición, persiguieron a los disidentes y anularon la democracia. Por el contrario, la seguridad democrática profundiza el 
pluralismo, protege por igual a todos los estamentos de la sociedad, y brinda todas las garantías y la protección en el proceso político, en particular en el electoral. El Gobierno, en lugar de recortar las libertades, so pretexto de enfrentar el terrorismo, ha aumentado el ejercicio práctico de aquéllas, el que se siente en la vida diaria. Lo ha hecho con una política que, en lugar de afectar los derechos humanos con la justificación de superar la inseguridad, todos los días está comprometida con su respeto, como condición de su propia legitimidad ${ }^{7}$.

Por lo tanto un primer momento de este análisis define en la conexión discursiva que se coloca como fundamento para conectar la realidad con un proyecto político, llevando a los ciudadanos a transformar sus perspectivas y haciendo de un elemento singular discursivo el fundamento homogenizante que dé linderos tanto a las acciones del Estado como a las percepciones e ilusiones del común de la sociedad. Esto significa ni más ni menos que trasponer y reducir las problemáticas de una sociedad a un discurso que se convierte en dinamizador y razón explicativa de dichas circunstancias. Creado el ambiente ideológico y discursivo, y siendo algo causal ¿cual es el paso a seguir?, cuyo objetivo será implementar y afirmar la idea política con resultados en gran manera visibles.

En segundo momento es necesario entender cómo se implementa y se calca en el imaginario publico resultados para legitimar los alcances del Estado sin observar los medios.

\section{1) Falsos Positivos}

En primera medida es importante revisar la "método" llamado "falsos positivos". En Octubre de 2009, los medios de comunicación revelan la desaparición de 14 jóvenes

\footnotetext{
7 Plan Nacional de Desarrollo 2006-2010 “Estado Comunitario: desarrollo para todos" [en línea] 2006, Disponible en la web: http://es.scribd.com/doc/44360/ESTADO-COMUNITARIO-DESARROLLO-PARA-TODOS
} 
desaparecidos en el municipio de Soacha ${ }^{8}$ ubicado al sur de la ciudad de Bogotá, sin que nadie diera razón de ellos, tiempo después estos jóvenes muertos son encontrados en la ciudad de Ocaña (Norte de Santander) al nororiente del país.

Las Fuerzas Militares de Colombia informa que estos jóvenes son miembros de las FARC y muertos en combate, después de indagar diferentes organismos de Derechos Humanos, la presión de los medios de comunicación y los testimonios de las madres de estos jóvenes, se divulga la noticia que estos jóvenes no pertenecían a ningún grupo al margen de la ley y son asesinados con el único fin de aumentar el conteo de muertos para agrandar los índices de eficiencia de la Política de Seguridad Democrática de Álvaro Uribe Vélez.

El Ministerio de Defensa al ver que la noticia con los argumentos que fueron asesinatos extrajudiciales o "falsos positivos" se expandía da la orden para que no sigan ocurriendo, es decir es un delito controlado, generando esto una mancha en la democracia colombiana que hasta el momento está quedando en la impunidad, pues en estos actos estuvieron involucradas 33 brigadas del Ejército Nacional y solo argumentó esta institución que los jóvenes fueron muertos en combate y el Ministerio de Defensa afirmando a su favor que "tanto el Estado como el Ministerio de Defensa no toleraría la violación de Derechos Humanos" (Juan Manuel Santos Ex ministro de Defensa, hoy candidato presidencial por el Partido de la $U$ ), a pesar de lo anterior el Ejercito seguía diciendo que estos jóvenes eran miembros de las FARC. Tiempo después cuando los resultados de las investigaciones confirman que los asesinatos son extrajudiciales, el gobierno acepta los falsos positivos y destituye a tres coroneles para un total de 26 militares destituidos 9 .

\footnotetext{
${ }^{8}$ Soacha es un municipio cercano a la capital del país, un municipio donde se cruzan todos los males de la sociedad, guerrilla, paramilitares, prostitución, bandas de ladrones, expendio de drogas y sobre todo un alto índice de pobreza.

${ }^{9}$ Morris, Hollman. "Contravia, Falsos positivos, una historia que se pudo evitar" [en línea] 2006. Disponible en la web: http://www.youtube.com/watch?v=TrFm7zVYD2Q
} 
Esto es el resumen de un numero de políticas equivocadas, por ejemplo el de exigir a los militares que sus meritos seria el número de muertos del enemigo es decir las FARC y no el de la eficiencia en el control de seguridad.

Algunos sectores de la opción afirma también que quienes reclutaron y mataron a estos jóvenes y de mas casos por falsos positivos eran los paramilitares en convivencia con las Fuerzas Militares, a esto se le suma el moldeamiento que le da el Estado a la opinión publica sobre este tema, pues se considera estos hechos como desaparición forzada, ejecuciones en estado de indefensión que no es otra cosa que crimines de Lesa Humanidad por parte del Estado. Aquí cabe la comparación de Chile y Argentina, mientras en estos países en años de dictadura ocurrieron desapariciones forzosas a causa de una ideología política, en este caso colombiano se hace premeditadamente por motivos de pobreza y motivos políticos independientemente si es opositor del gobierno.

La presión por resultados para ganar aceptación de las políticas de Seguridad Democrática, llevaron a implementar que entre mas bajas, mayores incentivos (se genera un Decreto de pago Recompensas), pero no hay un control si los muertos son en realidad guerrilleros pues todas las Fuerzas Militares se beneficiarían con los hechos.

Al desmovilizarse la mayor parte de las Autodefensas Militares de Colombia (AUC), los mandos medios se organizan de nuevos dando origen a grupos ilegales, uno de ellos llamados las "águilas negras", a estos se les acusa ser los directamente responsables del reclutamiento de los jóvenes, al darles bonificación (por el Decreto de Recompensas) por mostrarlos muertos en combate y a la vez el mando militar ganaba puntos en la opinión pública y la sociedad al demostrar que daba de baja aun a paramilitares siendo amigos de éstos. 
En el 2006 se dieron 235 casos de falsos positivos solo en el Departamento de Antioquia, donde se dan mayormente el número de casos en este Departamento, en total más de 1000 casos en el país de falsos positivos, un hecho digno de llevar a la Corte Penal Internacional, pues las madres de los jóvenes de Soacha fueron amenazadas de muerte por denunciar el caso.

A pesar de lo anterior, el 30 de diciembre de 2009 un Juez de la Republica amparado bajo la figura de vencimiento de términos deja en libertad a los militares involucrados en los asesinatos de los jóvenes de Soacha, esto demuestra que con la ilegalidad con que se legitima la violencia hay un gran índice de impunidad donde ya la sociedad colombiana no se inmuta por hechos que desde que Colombia nace como Nación ya 200 años se vienen generando. Se puede encontrar con lo anterior un vinculo de enlace con lo que afirma Johan Galtung cuando define "cultura de la violencia": "por "violencia cultural" nos referimos a aquellos aspectos de la cultura, la esfera simbólica de nuestra existencia ejemplificada por la religión y la ideología, el lenguaje y el arte, la ciencia empírica y la ciencia formal (lógica, matemática) - que puede ser utilizado para justificar o legitimar directa o estructural violencia"10

2) El tránsito de la guerra a la paz no es necesariamente es el tránsito de lo legal a lo ilegal

Haciendo un quiebre poco arbitrario, el año de 1982 se aprueba la ley general de amnistía en el mes de noviembre, derogando el Estatuto de Seguridad que en el gobierno de Turbay Ayala (1978-1982) confrontara de manera abierta no solo a los grupos armados sino también a organizaciones políticas y democráticas opositoras al gobierno. Esta ley general intentaba buscar un clima favorable en los diálogos de paz entre guerrilla y gobierno de Belisario Betancur.

\footnotetext{
${ }^{10}$ Galtung Johan "Cultural Violence" Journal of Peace Research, Vol 27, N 3 (Aug., 1990), pp. 291 disponible en la web: http://www.jstor.org/stable/423472
} 
Para el año 1984 el 28 de marzo se firma los acuerdos de cese al fuego y tregua, en el cual las partes firmantes se comprometían a un cese bilateral del fuego; sin embargo no aducía una entrega de armas por partes de las FARC. El resultado a esperar era encontrar mecanismos conjuntos para la salida política del conflicto, quienes firmaron el documento fueron una comisión de paz, dialogo y verificación como representantes del gobierno, directivos políticos y militares de las FARC-EP, la autodefensa obrera (ADO) y los destacamentos Simón Bolívar y Antonio Nariño del Ejercito de Liberación Nacional (ELN), poco tiempo después el M-19 y Ejército Popular de Liberación (EPL) firmaron el tratado, sin embargo el exterminio de algunos de sus militantes generaron una ruptura de los acuerdos.

Pese al acuerdo y el clima de negociación, los permanentes hostigamientos militares a los frentes guerrilleros, muestra como existía sino era una hipocresía por parte del gobierno en curso, una desconexión entre los objetivos militares y los objetivos del gobierno de Belisario Betancur, sin embargo las FARC decide en "unión con otros partidos democráticos de izquierda la lucha de las masas populares por el retorno a la normalidad, a la controversia civilizada, a una apertura democrática que garantice el libre ejercicio de la oposición y acceso a todos los medios de comunicación social, su organización, su lucha y movilización hacia crear un clima de participación popular en las gestiones del Estado".

Dando así inicio a lo que se conocería como la Unión Patriótica (UP), que como partido político en el debate electoral de 1986, elige 14 congresistas en el Senado y Cámara de Representantes, 18 diputados en 11 asambleas departamentales y 335 concejales en 187 concejos en el país. En las elecciones presidenciales obtienen 350.000 votos dos meses después, sin embargo su avance genero reacción de sectores opositores que empezaron a través de nexos con paramilitares a asesinar parlamentarios, diputados, concejales, militantes y simpatizantes del movimiento. 
Dichos nexos después de las investigaciones dejaron claro que quienes dinamizaron las desapariciones fueron miembros de inteligencia militar, aquí fallecieron candidatos presidenciales como Bernando Jaramillo Ossa, Jaime Pardo Leal y Carlos Pizarro.

Sin embargo las FARC-EP deciden continuar el diálogo y la negociación. En 1987 se constituye la Coordinadora Guerrillera Simón Bolívar que intenta agrupar la totalidad de insurgencia colombiana con el objetivo de aunarse en un proyecto común, adelantado procesos de paz ya bajo el gobierno de Virgilio Barco, donde el M-19, el PRT y un sector del EPL se incorporan a la vida civil, a pesar de estos la CGSB, en carta abierta sostiene que "la Asamblea Nacional Constituyente, debía contar con la presencia insurgente y con las de nuevas fuerzas políticas sociales, y subrayo que la imposición y exclusión generaría mas violencia". La respuesta fue el 9 de noviembre de 1990 mientras los colombianos elegían a los miembros de la Asamblea Nacional, el Ejército colombiano sin declaratoria de guerra lanzó un vasto operativo contra Casa Verde ${ }^{11}$, sede del Secretariado Nacional de las FARC, dando por terminado así cualquier intento de participación político de las FARC en el proceso histórico de la nueva Constitución colombiana en 1991.

\section{3) Palacio de Justicia}

El 6 de noviembre de 1985 a las 11:00 am, cuatro mujeres y tres hombres entran de civil, con papeles falsos al Palacio de Justicia ubicado en el centro de la ciudad de Bogotá ubicado al costad norte del Palacio de Nariño. 15 minutos más tarde Alfonso Jacquin verificada la seguridad real dentro de palacio llama a sus compañeros desde un teléfono público y cinco minutos más tarde llegan dos vehículos con 28 guerrilleros del M-19

\footnotetext{
${ }^{11}$ La Operación Casa Verde, llamada oficialmente Operación Colombia, fue una operación militar perpetrada por las Fuerzas Militares de Colombia, siendo presidente de Colombia César Gaviria Trujillo, contra los campamentos de Casa Verde de la guerrilla de las Fuerzas Armadas Revolucionarias de Colombia (FARC) el 9 de diciembre de 1990. Tropas de la Séptima Brigada del Ejército de Colombia atacaron el área que cubre el río Duda, quebrada las Mil, las estribaciones de la Cordillera Oriental en el municipio de La Uribe en el departamento del Meta, con el fin de desmantelar el Estado Mayor y el Secretariado de las FARC.
} 
rompiendo la puerta del sótano del palacio y enlistando posiciones. Dentro del edificio se organiza dos unidades del M-19, la primera dirigida por Andrés Almarales y Ariel Sánchez, debería tomar la parte posterior del edificio y la otra dirigida por Luis Otero, Alfonso Jacquin y Elbencio Ruiz el resto del palacio. Los negociadores serian Andrés Almarales y Alfonso Jacquin. En el momento efectivo de la toma la guerrilla pierde a dos de sus integrantes pero abate a seis personas con intercambios de disparos con escoltas de magistrados. Diez minutos después cuando el M-19 ha asegurado el edificio llegan diez carros armados y tanques blindados a la Plaza de Bolívar y se dan dos días, en los cuales la institucionalidad busca restaurar el orden a partir de negociaciones frente al Ejercito que intenta reponer su orgullo militar venido a menos por acciones militares efectuadas por el M-19 que habían dejado la institución militar aun en vergüenza (recuérdese el robo de armas al Canton Norte por parte del M-19 sin un solo disparo). Los hechos ocurridos entre estos dos días han sido objeto de análisis no solo judicial sino político ya que el papel de las Fuerzas Militares fue más allá de recuperar el edificio, según la Comisión de la Verdad el Presidente Belisario Betancourt admitió que los altos comandantes del Ejercito actuó por fuera de las disposiciones del Ejecutivo, es decir fue un golpe de Estado en busca de obtener un fin supremo recuperar una institución; la Fuerza Armada desestimó las acciones y negociaciones del Ejecutivo para recuperar el orden y uso de su monopolio de la violencia para cometer hechos por fuera de la legalidad, tanto así que la retoma al Palacio, la desaparición de civiles y la muerte de algunos magistrados, incineración de cuerpos, quema de Palacio y archivos que allí se encontraban, desaparición de pruebas materiales y la obstaculización de la justicia. Veinte años después de los hechos muestran el desmedido uso de la fuerza como causa de dichos actos delictivos.

Una de los principales conclusiones del informe de la Comisión es que reconoce que se cometieron delito de lesa humanidad, durante la retoma del Palacio y con posterioridad a ésta por parte de agentes del Estado, por lo tanto tales delitos no son imprescriptibles. Pero, su investigación y sanción le corresponde a instancias judiciales como la Fiscalia, porque la Comisión no es un organismo público y no tiene la facultad o potestad para dictar sentencias judiciales y hacerlas cumplir. 
La Comisión analizó la legitimidad de las acciones de los organismos del Estado, desde el punto de vista de que todo Estado democrático debe respetar los derechos fundamentales de las personas $y$, que las acciones para enfrentar ataques subversivos deben guardar proporcionalidad y tratar de preservar la vida de los civiles en medio del fuego.

El informe afirma con seguridad que existieron desaparecidos y logró saber quiénes eran, pero no descarta que el número pueda ser mayor, porque aún hace falta ubicar los restos, punto en el cual la Comisión desea recabar, pero este es un ejercicio muy sensible, aunque existe información valiosa al respecto. El aparato de justicia a pesar de contar con los indicios suficientes, los testimonios sobre este tema, y la capacidad institucional no logró en tantos años algo tan puntual como lo asegurado por el informe preliminar. Es claro que está situación no obedeció a la incapacidad del aparato judicial sino a las barreras que existían.

En el informe preliminar están incluidas las conclusiones más importantes de las indagaciones realizadas por la Comisión, pero el informe final contendrá las pruebas de las afirmaciones que realizan, sin embargo, en términos generales sus resultados no van a variar en gran manera. En cuanto al tema de los desaparecidos la Comisión espera continuar sus indagaciones porque es posible realizar avances muy importantes y aún faltan muchas preguntas por responder. La fecha de publicación del informe final no está definida aún, debido a problemas de carácter logístico, temporal y económico, que han dificultado el trabajo de la Comisión.

A pesar de los linderos marcados para la labor de la Comisión afirma que: "es muy importante sí la Fiscalía utiliza más el informe de la Comisión y las pruebas, que nosotros no las llamamos pruebas, las versiones de lo que tenemos, porque es claro que esclarece en algunos puntos muy importantes" (Herrera 2007). Significa esto que el informe de la Comisión posee información y material relevante para las acciones judiciales que está 
adelantando la Fiscalía y que han dado resultados cercanos como la orden de captura sobre el Coronel (R) Luis Alfonso Plazas, y la vinculación del General (R) Arias Cabrales a las investigaciones por desaparición forzada y tortura, de personas que fueron rescatadas con vida del Palacio.

Sin embargo, aún hay que recorrer un largo sendero para esclarecer la verdad, la cual, como señala el Informe Preliminar es "el derecho fundamental de las víctimas, de los perjudicados y de la sociedad a quienes los responsables deben pedir perdón, como también han de hacerlo todos los indolentes [...], por su indiferencia y la propensión a tolerar la impunidad" (Informe Preliminar, pp. 55-56). Una vez pueda saberse a quiénes perdonar y una vez haya justicia y reparación material por autores, partícipes y el Estado podrá abrirse el camino hacia la reconciliación. La sociedad, señala el Informe Preliminar, "siempre reclamará conocer quiénes, cómo y por qué han agredido a alguno o algunos de sus miembros" (Informe Preliminar, p. 56) y mientras esto no sea esclarecido no podrá haber ni paz ni reconciliación.

\section{A manera de conclusiones}

Hecho un recorrido, a propósito indiscriminado, donde se transitó por tres momentos históricos disimiles a saber: La Toma del Palacio de Justicia, La Operación Casa Verde y los mal llamados Falsos Positivos que no son sino ejecuciones extrajudiciales, se quiso poner de presente no solo que el Estado colombiano es un actor generador y perpetuador de la violencia, sino que el uso de acciones que traspasan los límites de lo legal hacen parte de la construcción histórica del conflicto colombiano y dicha ilegalidad responde a ordenes internos en donde por encima de cualquier valor los fines deben ser alcanzados de manera contundente haciendo lo legal agua en el espacio de los medios para dicho objetivo. Constitucionalmente el Estado colombiano es un Estado de Derecho, sin embargo, tal limitación normativa no es un objeto eficaz para las acciones del Estado. 
No es optimo hacer una relación proporcional o directa entre el uso de la institucionalidad y la violencia en momentos diferenciados, es decir violencia política y doméstica, pero a partir de lo observado y de los datos documentados es fácil observar como la violencia se constituye en el elemento dinamizador de los conflictos en las esferas sociales, se reproduce de manera micro y macro dando cada vez más espacios a que en el centro de la conciencia colombiana se de la banalización de la violencia y se use de esta como instrumento eficaz para la resolución de cualquier diferencia, ya sea en el ámbito familiar como en lo estatal.

Pero en este lugar el aporte de lo macro es determinante, es decir consciente y proyectado, cuando en un discurso político institucional se enuncian sentidos en los cuales el enfrentamiento es la salida para finalizar con la violencia política y de allí con la otredad, y a partir de esto se implementan acciones para sustentar dichos preceptos, poco a poco deben mostrarse resultados que sostengan en el imaginario público, pero cuando los resultados son logrados a partir de acciones ilegales por parte del actor de quien no debería usar tales prácticas, se rompen los valores con principios institucionalizados, es decir no hay referentes que muestren al Estado Colombiano como un lugar en el cual los pactos sociales y políticos se respeten y allí aparece la banalidad, sin embargo esa condición no es única del gobierno actual, está claramente observada en momentos disimiles históricos analizados en este trabajo que lo acción ilegal hace parte del concurso de opciones a las cuales el Estado ha recurrido con frecuencia.

La respuesta de la sociedad está enmarcada por dos momentos uno es el rechazo a cualquier acto de violencia venga de donde venga, sin embargo eso sería un deber ser, las marchas por la paz en Colombia y aun en el exterior se convirtieron en manifestaciones de una mirada a la situación colombiana, eran legitimas siempre y cuando apoyaran la institucionalidad, el despliegue mediático fue eficaz y así la concurrencia, sin embrago en el momento en que las víctimas del conflicto y sobretodo del Estado y de los paramilitares decidieron marchar, las amenazas contra organizadores, el discurso patriotero desvirtuando tal ejercicio y vinculándolo con el otro extremo es decir 
con las FARC. Obviamente la marcha no fue tan exitosa como aquella que la institucionalidad apoyaba.

Podrían nombrarse muchos casos que fortalezcan esta observación y no se está con este documento descubriendo el agua tibia, pero lo que sí se puede dejar a manera de reflexión es que mientras un Estado se salga de los parámetros en los que legalmente pueda actuar, los índices de violencia no solo se multiplican sino construyen en el imaginario público la sensación de que hay muchos medios no necesariamente positivos que posibilitan cualquier fin, banalizar, es colocar en este momento una relación entre costos y beneficios, es una razón precisa que explica que en Colombia la cultura de la Violencia se sustenta por el acto ilegal como medio necesario que genera bajos costos frente a los supuestos beneficios que traen dichas acciones y cuando esto viene del Estado se radicaliza un quiebre ético tanto en lo institucional como en la sociedad, de allí que algunos actos dados en el gobierno actual sean tomado como mínimos frente a las pírricas glorias de tal administración. Solo es esperar que el papel de la justicia colombiana haga su curso y que a pesar del $90 \%$ de índice de impunidad no se aplique para las nefastas acciones del gobierno entre el 2002 y el 2010.

Si queremos cauterizar las heridas debemos enfrentar el hecho paradójico de que si el pasado continua atormentando a Ruanda, es precisamente porque no es pasado. El mayor obstáculo en el camino de la reconciliación es el deseo de venganza. La venganza ayuda a conservar los vínculos que unen a las generaciones, y la violencia que engendra es una forma ritual de respeto por los muertos, en los que la comunidad basa su legitimidad. Reconciliarse significa romper la espiral de la venganza intergeneracional. La reconciliación puede romper el círculo de la venganza solo a condición que se respeten los muertos. Sin apología, sin reconocimiento de los hechos, el pasado nunca vuelve a su puesto 
Si queremos cauterizar las heridas del conflicto debemos enfrentar el hecho paradójico de que si el pasado continua atormentando a los colombianos, es precisamente porque no es pasado, el mayor obstáculo en el camino de la reconciliación es el deseo de venganza que se ha gestado desde los inicios del conflicto colombiano. La venganza ayuda a conservar los vínculos que unen a las generaciones, y la violencia que engendra junto con la ilegalidad es una forma ritual de respeto por los muertos, en que también la sociedad basa su legitimidad junto con el narcotráfico, las pandillas, la insurgencia es hoy para el colombiano una salida fácil a los problemas económicos y sociales que enfrenta. Por lo tanto reconciliarse significa romper esa cultura de violencia siendo la reconciliación un método en las negociaciones de paz, pues sin apología, sin reconocimiento de los hechos, el pasado nunca vuelve a su puesto. Sin embargo para Waltz en su libro "el hombre, el Estado y la guerra" la naturaleza humana está intrínsecamente relacionada de otros factores, como la socialización como explicación de la personalidad del comportamiento humano, es decir esta primera imagen de Waltz no es la única para elaborar una teoría sobre las causas de la guerra, así tampoco podría decir que en Colombia la naturaleza del conflicto prima por la cultura de violencia que muchos autores interpretan para su explicación, pues es tan solo una herramienta de análisis y que se debe observar los procesos negociaciones de paz en la escuela racionalista como Jon Elster donde afirma que: "la sumisión autoritaria a los deseos de otro sujeto con el cual se guardan permanentes relaciones de interacción, suele ser una eficiente forma de resolver lo que los teóricos llaman "disonancia cognoscitiva", es decir la violencia, en fin, es otra forma muy recurrida para resolver conflictos, en otras palabras cuando los intereses del conflicto son mayores que en estado de paz, la violencia o la guerra continua. 


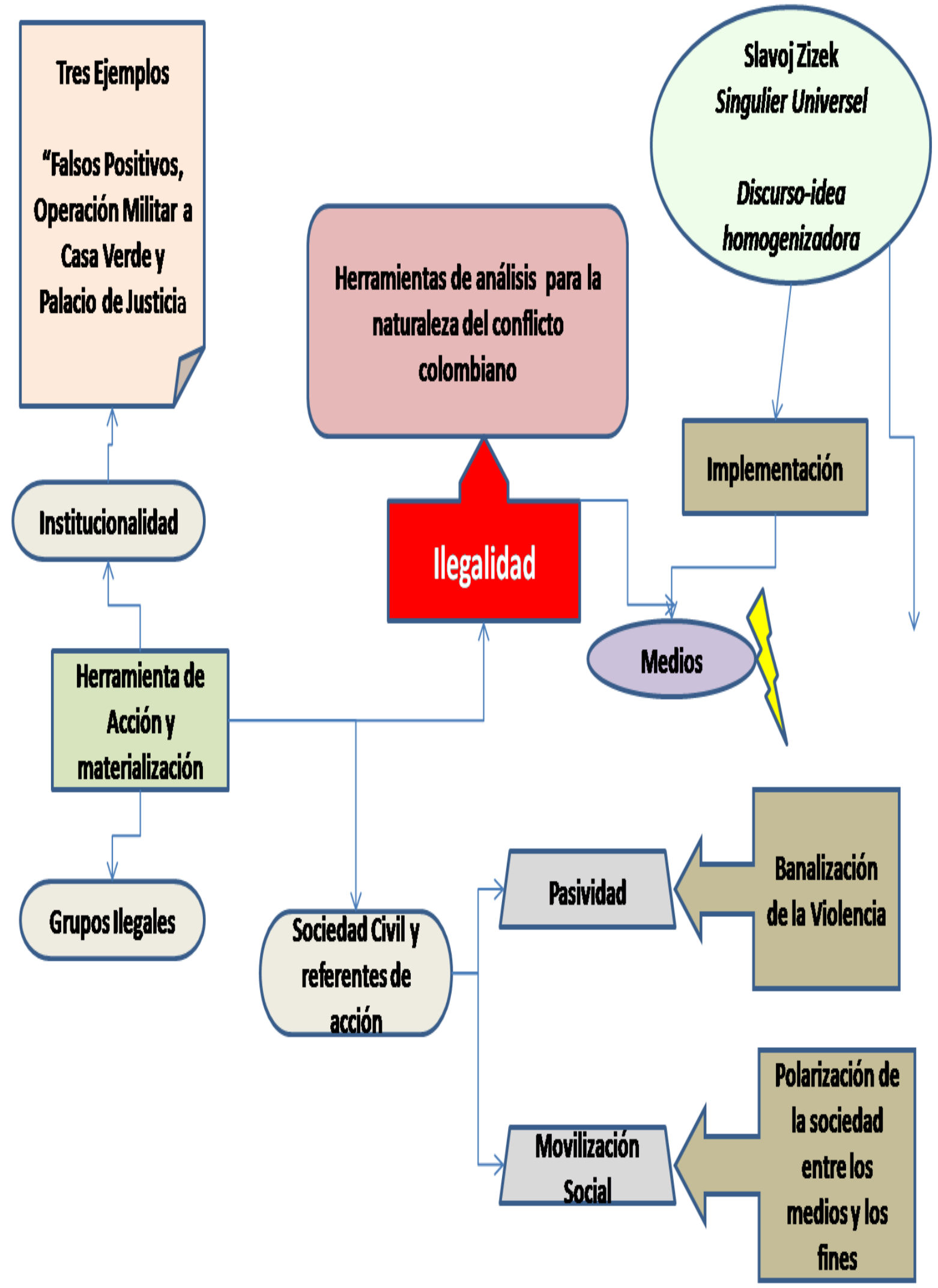




\section{BIBLIOGRAFIA}

- ABELLO Ignacio, "violencias y culturas: seguido de dos estudios sobre Nietzsche y Foucault, a propósito del mismo tema" CESO, Universidad de los Andes, Enero 2003.

- PECAUT Daniel, “guerra contra la sociedad”, Editorial Planeta, 2001.

- RIZO Otero Harold José, "Evolución del conflicto armado en Colombia e Iberoamerica" Editorial Nomos, 2002.

- BATTALEME Juan, "América del Sur: Conflicto y Cooperación en la dinámica Regional", Agenda Internacional, Año 3, Vol. 9, Julio - Septiembre 2006

- MAYA Maureen, Petro Gustavo, "Prohibido olvidar: dos miradas sobre la toma del Palacio de Justicia".

- GALTUNG Johan, "Cultural Violence" Journal of Peace Research, Vol 27, $\mathrm{N}^{\circ} 3$ (Aug., 1990), pp. 291-305.

- GALTUNG Johan, “Sobre la paz”, Barcelona, Editorial Fontamara, 1985.

- WALTZ Kenneth, "El hombre, el Estado y la guerra” Buenos Aires, Editorial Nova 1970.

- VELÁSQUEZ Natalia Eugenia, "violencia como categoría de identidad personal y cultural en Colombia" Universidad Jesuita Alberto Hurtado, Ponencia, I Encuentro Latinoamericano de Estudiantes de Posgrado en Ciencias Sociales.

- COBO Borda Juan Gustavo, "Colombia: cultura y violencia" http://www.cervantesvirtual.com/servlet/SirveObras/91338365214911274532279/209774_ 0021.pdf 